\title{
Analysis of strip electric saturation model of crack problem in piezoelectric materials
}

\author{
T.C. Wang \\ LNM, Institute of Mechanics, Chinese Academy of Sciences, 15 Zhong Guan Cun Road, Beijing 100080, People's Republic of China
}

Received 21 April 1998; in revised form 7 September 1999

\begin{abstract}
This paper presents a fully anisotropic analysis of strip electric saturation model proposed by Gao et al. (1997) (Gao, H.J., Zhang, T.Y., Tong, P., 1997. Local and global energy release rates for an electrically yielded crack in a piezoelectric ceramic. J. Mech. Phys. Solids, 45, 491-510) for piezoelectric materials. The relationship between the size of the strip saturation zone ahead of a crack tip and the applied electric displacement field is established. It is revealed that the critical fracture stresses for a crack perpendicular to the poling axis is linearly decreased with the increase of the positive applied electric field and increases linearly with the increase of the negative applied electric field. For a crack parallel to the poling axis, the failure stress is not effected by the parallel applied electric field. In order to analyse the existed experimental results, the stress fields ahead of the tip of an elliptic notch in an infinite piezoelectric solid are calculated. The critical maximum stress criterion is adopted for determining the fracture stresses under different remote electric displacement fields. The present analysis indicates that the crack initiation and propagation from the tip of a sharp elliptic notch could be aided or impeded by an electric displacement field depending on the field direction. The fracture stress predicted by the present analysis is consistent with the experimental data given by Park and Sun (1995) (Park, S., Sun, C.T., 1995. Fracture criteria for piezoelectric materials. J. Am. Ceram. Soc 78, 1475-1480). (C) 2000 Elsevier Science Ltd. All rights reserved.
\end{abstract}

Keywords: Piezoelectric materials; Crack problem; Strip saturation

\section{Introduction}

Piezoelectric materials have been extensively used in smart devices as sensors and actuators. The combined mechanical and electrical loads give rise to sufficiently high stresses in these devices which result in catastrophic failure. The fracture mechanics of piezoelectric materials have attracted many theoretical studies (Parton, 1976; Deeg, 1980; Pak and Herrmann, 1986; McMeeking, 1989; Pak, 1990;

E-mail address: tcwang@cc5.imech.ac.cn (T.C. Wang).

0020-7683/00/\$ - see front matter (C) 2000 Elsevier Science Ltd. All rights reserved.

PII: S0020-7683(99)00255-3 
Sosa, 1992; Suo et al., 1992; Suo, 1993; Zhang and Hack, 1992; Yang and Suo, 1994; Dunn, 1994; Zhang and Tong, 1996 among others). But there are remarkable discrepancies between theory and experiments (see Pak and Tobin, 1993; Park and Sun, 1995; Kumar and Singh, 1996). The theoretical studies (Pak and Tobin, 1993) have shown that the applied electric field should inhibit crack propagation irrespective of its sign. Park and Sun (1995) measured the failure stresses of cracks perpendicular to the poling axis in compact tension and three point bend specimens made of PZT-4 ceramics and found that the failure stresses are decreased with the increase of the positive applied electric field (in the same direction as the poling axis) and increased with the increase of the applied negative electric field. They argued that the fracture process of ceramic materials is a pure mechanical process and should be controlled only by the mechanical part of the energy release rate.

A strip electric saturation model has been proposed by Gao et al. (1997) in order to explain the effects of the applied electric field on the failure stress of piezoelectric materials. This model can be considered as a generalization of the classical Dugdale model in fracture mechanics. They thought the piezoelectric ceramics such as lead zirconate titanate $(\mathrm{PZT})$ and barium titanate $\left(\mathrm{BaTiO}_{3}\right)$ can be considered as brittle materials and plastic yielding is rather difficult for theses materials. But theses materials are electrically more ductile. The plastic yielding zone ahead of the crack tip is much smaller than the electric saturation zone. Hence one should take into account the effects of the electric saturation and neglect the effects of the plastic yielding. Gao et al. (1997) found that the local energy release rate gives reasonable prediction which broadly agrees well with the experimental results. But the analysis given by Gao et al. (1997) is based on a simplified electroelasticity formulation.

A fully anisotropic analysis of the strip electric saturation model for piezoelectric materials is presented in this paper. The complete coupling between the mechanical and electrical behaviors is taken into account. The relationship between the size of the strip saturation zone ahead of a crack tip and the applied electric field is established. It is revealed that the critical fracture stresses for a crack perpendicular to the poling axis is linearly decreased with the increase of the positive applied electric field and increases linearly with the increase of the negative applied electric field. For a crack parallel to the poling axis, the failure stress is not effected by the parallel applied electric field.

\section{Basic formulas}

The constitutive equations for piezoelectric materials are

$$
\begin{gathered}
\sigma_{i j}=c_{i j k l} \gamma_{k l}-e_{k i j} E_{k} \\
D_{i}=e_{i k l} \gamma_{k l}+\varepsilon_{i k} E_{k}
\end{gathered}
$$

where $\sigma_{i j}, \gamma_{i j}$ are the stress tensor and strain tensor respectively. $D_{i}, E_{i}$ are the electric displacement and electric field, respectively, $c_{i j k l}, e_{i k l}$, and $\varepsilon_{i j}$ are the elastic, piezoelectric and dielectric constants, respectively. The mechanical and electrical equilibrium equations take the form

$$
\begin{aligned}
\sigma_{i j, i} & =0 \\
D_{i, i} & =0
\end{aligned}
$$

Strain $\gamma_{i j}$, electric field $E_{i}$ can be expressed as 


$$
\begin{aligned}
\gamma_{i j} & =\frac{1}{2}\left(u_{i, j}+u_{j, i}\right) \\
E_{i} & =-\phi_{, i}
\end{aligned}
$$

where $u_{i}$ is displacement, and $\phi$ is the electric potential.

Substituting Eqs. (1) and (3) into Eq. (2), one obtain

$$
\begin{aligned}
& \left(c_{i j k l} u_{k}+e_{l i j} \phi\right)_{, l i}=0 \\
& \left(e_{i k l} u_{k}-\varepsilon_{i l} \phi\right)_{, l i}=0
\end{aligned}
$$

Consider two-dimensional problem, the general solution can be expressed by complex potential

$$
\left\{u_{i}, \phi\right\}=\boldsymbol{a} f\left(\zeta_{1} x+\zeta_{2} y\right)
$$

where $\boldsymbol{a}$ is a four-component column. $\zeta_{1}=1, \zeta_{2}=p$, Substituting Eq. (5) into Eq. (4), it follows

$$
\begin{aligned}
& \left(c_{\alpha j k \beta} a_{k}+e_{\alpha \beta j} a_{4}\right) \zeta_{\alpha} \zeta_{\beta}=0 \\
& \left(e_{\alpha \beta k} a_{k}-\varepsilon_{\alpha \beta} a_{4}\right) \zeta_{\alpha} \zeta_{\beta}=0
\end{aligned}
$$

where $\alpha, \beta$ take on the values 1 and $2 . j, k$ have the values 1,2 and 3 . This is an eigen problem for column $\boldsymbol{a}$. Suo et al. (1992) have shown that the eigenequation of this problem has eight complex roots form four conjugate pairs. Let $p_{1}, p_{2}, p_{3}, p_{4}$ be the four roots with positive imaginary part. We have

$$
\left\{u_{i}, \phi\right\}=2 \operatorname{Re} \sum_{K=1}^{4} \boldsymbol{a}_{K} f_{K}\left(z_{K}\right)
$$

where $z_{K}=x+p_{K} y$.

For the stress and electric displacement components, one obtains

$$
\begin{aligned}
& \left\{\sigma_{2 j}, D_{2}\right\}=2 R e \sum_{K=1}^{4} \boldsymbol{b}_{K} f_{K}^{\prime}\left(z_{K}\right) \\
& \left\{\sigma_{1 j}, D_{1}\right\}=-2 \operatorname{Re} \sum_{K=1}^{4} \boldsymbol{b}_{K} p_{K} f_{K}^{\prime}\left(z_{K}\right)
\end{aligned}
$$

Column $\boldsymbol{b}$ has the components

$$
\begin{aligned}
& b_{j}=\left(c_{2 j k 1} a_{k}+e_{12 j} a_{4}\right)+\left(c_{2 j k 2} a_{k}+e_{22 j} a_{4}\right) p \\
& b_{4}=\left(e_{21 k} a_{k}-\varepsilon_{21} a_{4}\right)+\left(e_{22 k} a_{k}-\varepsilon_{22} a_{4}\right) p,
\end{aligned}
$$

Introducing $4 \times 4$ matrices $\boldsymbol{A}$ and $\boldsymbol{B}$

$$
A=\left[a_{1}, a_{2}, a_{3}, a_{4}\right], \quad B=\left[b_{1}, b_{2}, b_{3}, b_{4}\right]
$$

Define a function vector $f(z)$ of a single variable 


$$
\boldsymbol{f}(z)=\left\{f_{1}(z), f_{2}(z), f_{3}(z), f_{4}(z)\right\}
$$

Then the generalized displacement and the traction on the real axis can be expressed as

$$
\begin{aligned}
& \boldsymbol{U}(x)=\left\{u_{j}, \phi\right\}=\boldsymbol{A} \boldsymbol{f}(x)+\overline{\boldsymbol{A}} \overline{\boldsymbol{f}(x)} \\
& \boldsymbol{t}(x)=\left\{\sigma_{2 j}, D_{2}\right\}=\boldsymbol{B} \boldsymbol{f}^{\prime}(x)+\overline{\boldsymbol{B}} \overline{\boldsymbol{f}^{\prime}(x)}
\end{aligned}
$$

\section{Strip electric saturation model}

\subsection{Mechanics analysis}

Fig. 1 shows a finite crack of length $2 a$ in an infinite piezoelectric plate subjected to remote stresses $\sigma_{11}^{\infty}, \sigma_{22}^{\infty}$ and electric displacements $D_{1}^{\infty}, D_{2}^{\infty}$ loadings. The crack lies on the $x$-axis. Ahead of the crack tip, there is a strip electric saturation zone. On the crack faces, the generalized traction should vanish

$$
\sigma_{22}=0, \quad \tau_{21}=0, \quad \tau_{23}=0, \quad D_{2}=0, \quad|x| \leq a
$$

In the strip electric saturation zone, we have

$$
u_{i}^{+}=u_{i}^{-}, \quad \sigma_{2 i}^{+}=\sigma_{2 i}^{-}, \quad D_{2}=D_{s}, \quad a \leq|x| \leq c
$$

The homogeneous fields produced by the applied mechanical and electrical fields are

$$
\begin{aligned}
& \sigma_{11}=\sigma_{11}^{\infty}, \quad \sigma_{22}=\sigma_{22}^{\infty}, \quad \tau_{12}=\tau_{13}=\tau_{23}=0, \\
& D_{1}=D_{1}^{\infty}, \quad D_{2}=D_{2}^{\infty}
\end{aligned}
$$

The inhomogeneous fields induced by the crack should satisfy the following boundary conditions on the crack faces

$$
\begin{aligned}
& \boldsymbol{t}^{+}(x)=\boldsymbol{t}^{-}(x)=-\boldsymbol{T}, \quad|x|<a \\
& \boldsymbol{T}=\left\{0, \sigma_{22}^{\infty}, 0, D_{2}^{\infty}\right\}
\end{aligned}
$$

On the strip electric saturation zone, we have

$$
\boldsymbol{t}^{+}(x)=\boldsymbol{t}^{-}(x)
$$

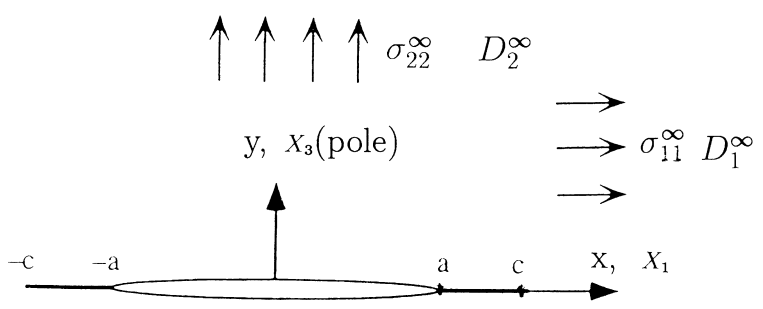

Fig. 1. An electric saturation crack perpendicular to the poling axis. 


$$
\begin{aligned}
& u_{i}^{+}=u_{i}^{-}, \quad a \leq|x| \leq c, \quad i=1,2,3 \\
& D_{2}^{+}=D_{2}^{-}=D_{s}-D_{2}^{\infty}
\end{aligned}
$$

The continuity of $\boldsymbol{t}(x)$ on the whole real axis implies that

$$
\boldsymbol{B} \boldsymbol{f}^{\prime+}(x)+\overline{\boldsymbol{B}} \overline{\boldsymbol{f}}^{\prime-}(x)=\boldsymbol{B} \boldsymbol{f}^{\prime-}(x)+\overline{\boldsymbol{B}} \overline{\boldsymbol{f}}^{\prime+}(x), \quad-\infty<x<+\infty .
$$

From above equation, Suo et al. (1992) have shown that

$$
\boldsymbol{h}(z)=\boldsymbol{B} \boldsymbol{f}^{\prime}(z)=\overline{\boldsymbol{B}} \overline{\boldsymbol{f}}^{\prime}(z)
$$

where $\boldsymbol{f}(z)$ is the complex function vector of the inhomogeneous field. This complex function vector $\boldsymbol{h}(z)$ should satisfy the boundary condition (19) along the crack faces,

$$
\boldsymbol{h}^{+}(x)+\boldsymbol{h}^{-}(x)=-\boldsymbol{T}, \quad|x|<a
$$

On the other hand, we have

$$
i \boldsymbol{\delta}^{\prime}(x)=\boldsymbol{H} \boldsymbol{B} \boldsymbol{f}^{\prime+}(x)-\boldsymbol{H} \boldsymbol{B} \boldsymbol{f}^{\prime-}(x)
$$

where $\boldsymbol{\delta}^{\prime}(x)=\left\{u_{1}^{+}-u_{1}^{-}, u_{2}^{+}-u_{2}^{-}, u_{3}^{+}-u_{3}^{-}, \phi^{+}-\phi^{-}\right\}$is the generalized opening displacement. $\boldsymbol{H}$ is a real matrix

$$
\boldsymbol{H}=2 \operatorname{Re}\left\{i \boldsymbol{A} \boldsymbol{B}^{-1}\right\}
$$

Introduce a new complex function vector

$$
\boldsymbol{g}(z)=\boldsymbol{H B} f^{\prime}(z)
$$

From Eq. (22), one can see that the component functions $g_{i}(z), i=1,2,3$ are holomorphic in whole $z$ plane with a cut $(-a, a)$. The component function $g_{4}(z)$ is a holomorphic function in whole $z$ plane with a cut $(-c, c)$. From Eq. (28), one obtains

$$
\boldsymbol{h}(z)=\boldsymbol{H}^{-1} \boldsymbol{g}(z)=\boldsymbol{\Lambda} \boldsymbol{g}(z)
$$

where

$$
\boldsymbol{\Lambda}=\boldsymbol{H}^{-1} .
$$

Eq. (25) can be represented in its component form,

$$
\begin{aligned}
& \Lambda_{i k}\left(g_{k}^{+}(x)+g_{k}^{-}(x)\right)+\Lambda_{i 4}\left(g_{4}^{+}(x)+g_{4}^{-}(x)\right)=-T_{i}, \quad i=1,2,3,|x|<a \\
& \Lambda_{4 k}\left(g_{k}^{+}(x)+g_{k}^{-}(x)\right)+\Lambda_{44}\left(g_{4}^{+}(x)+g_{4}^{-}(x)\right)=-T_{4}, \quad|x|<a
\end{aligned}
$$

Eliminating $g_{4}^{+}(x)+g_{4}^{-}(x)$ from Eqs. (31) and (32), one obtains

$$
\Lambda_{i k}^{*}\left(g_{k}^{+}(x)+g_{k}^{-}(x)\right)=-T_{i}^{*}, \quad|x|<a
$$

where

$$
\Lambda_{i k}^{*}=\Lambda_{i k}-\Lambda_{i 4} \Lambda_{4 k} / \Lambda_{44},
$$




$$
T_{i}^{*}=T_{i}-T_{4} \Lambda_{i 4} / \Lambda_{44}, \quad i, k=1,2,3
$$

Eq. (33) can be rewritten in vector form as,

$$
\boldsymbol{\Lambda}^{*} \boldsymbol{g}^{*+}(x)+\boldsymbol{\Lambda}^{*} \boldsymbol{g}^{*-}(x)=-\boldsymbol{T}^{*}, \quad|x|<a
$$

where $\boldsymbol{\Lambda}^{*}$ is $3 \times 3$ matrix, the elements are $\Lambda^{* i j} \cdot \boldsymbol{g}^{*}, \boldsymbol{T}^{*}$ are two three-component columns.

$$
\begin{aligned}
& \boldsymbol{g}^{*}(z)=\left\{g_{1}(z), g_{2}(z), g_{3}(z)\right\} \\
& \boldsymbol{T}^{*}=\left\{T_{1}^{*}, T_{2}^{*}, T_{3}^{*}\right\}
\end{aligned}
$$

Obviously the function vector $\boldsymbol{g}^{*}(z)$ is holomorphic in whole $z$ plane except cut $L$. From Eq. (35), one obtains

$$
\begin{aligned}
& \boldsymbol{\Lambda}^{*} \boldsymbol{g}^{*}(z)=\boldsymbol{T}^{*} f_{0}^{\prime}(z) \\
& f_{0}^{\prime}(z)=\frac{1}{2}\left\{\frac{z}{\sqrt{z^{2}-a^{2}}}-1\right\}
\end{aligned}
$$

From Eqs. (23), (28) and (32), it follows,

$$
\begin{aligned}
& g_{4}^{+}(x)+g_{4}^{-}(x)=-\left\{\Lambda_{4 k}\left[g_{k}^{+}(x)+g_{k}^{-}(x)\right]+T_{4}\right\} \Lambda_{44}, \quad|x|<a \\
& g_{4}^{+}(x)+g_{4}^{-}(x)=-\left\{\Lambda_{4 k}\left[\left(g_{k}^{+}(x)+g_{k}^{-}(x)\right]+T_{4}\right\} / \Lambda_{44}+D_{s} / \Lambda_{44} \quad a \leq|x| \leq c\right.
\end{aligned}
$$

The solution of Eq. (40) is

$$
g_{4}(z)=\left\{-\Lambda_{4 k} g_{k}(z)+T_{4} f_{c}^{\prime}(z)\right\} / \Lambda_{44}+D_{s} g_{0}(z) / \Lambda_{44}
$$

where

$$
\begin{aligned}
& f_{c}^{\prime}(z)=\frac{1}{2}\left[\frac{z}{\sqrt{z^{2}-c^{2}}}-1\right] \\
& g_{0}(z)=\frac{1}{\pi}\left\{\frac{\pi}{2}-\frac{1}{2 i} \log \frac{\frac{z}{a} \cdot \frac{\sqrt{c^{2}-a^{2}}}{\sqrt{z^{2}-c^{2}}}+i}{\frac{z}{a} \cdot \frac{z}{\sqrt{c^{2}-a^{2}}}-i}-\frac{z}{\sqrt{z^{2}-c^{2}}} \arccos \left(\frac{a}{c}\right)\right\},
\end{aligned}
$$

It can be easily proved that the function $g_{0}(z)$ is holomorphic in whole $z$ plane outside the cut $(-c, c)$. The complex function $g_{0}(z)$ has the following behaviors,

$$
\begin{array}{ll}
g_{0}^{+}(x)+g_{0}^{-}(x)=0, & |x|<a \\
g_{0}^{+}(x)+g_{0}^{-}(x)=1, & a \leq|x| \leq c
\end{array}
$$

The function $g_{0}(z)$ vanishes at infinite. The function $\log \frac{\zeta+i}{\zeta-i}$ is calculated according to Fig. 2. 


$$
\log \frac{\zeta+i}{\zeta-i}=\log \frac{r_{2}}{r_{1}}+i\left(\theta_{2}-\theta_{1}\right)
$$

where $r_{1}$ and $r_{2}$ are the amplitudes of the complex variables $\zeta+i$ and $\zeta-i$, respectively. $\theta_{1}$ and $\theta_{2}$ are the inclined angles of the complex variables $\zeta+i$ and $\zeta-i$ with respect to the negative imaginary axis. Eqs. (38) and (41) provide the complete solution of $\boldsymbol{g}(z)$.

In order to evaluate the size of the strip electric saturation zone ahead of the crack tip, let us consider the traction ahead of the electric saturation zone. We have

$$
\begin{aligned}
& \boldsymbol{t}(x)=\left\{\sigma_{21}, \sigma_{22}, \sigma_{23}, D_{2}\right\}=\boldsymbol{B} \boldsymbol{f}^{\prime+}(x)+\boldsymbol{B} \boldsymbol{f}^{\prime}-(x)=\boldsymbol{\Lambda} \boldsymbol{g}^{+}(x)+\Lambda g^{-}(x), \quad|x|>c \\
& D_{2}=\Lambda_{4 k}\left(g_{k}^{+}(x)+g_{k}^{-}(x)\right)+\Lambda_{44}\left(g_{4}^{+}(x)+g_{4}^{-}(x)\right) \\
& =2 T_{4} f_{c}^{\prime}(x)+D_{s}\left(g_{0}^{+}(x)+g_{0}^{-}(x)\right) \\
& =\left(D_{2}^{\infty}-\frac{2}{\pi} D_{s} \arccos \left(\frac{a}{c}\right)\right) \frac{x}{\sqrt{x^{2}-c^{2}}}-D_{2}^{\infty}+D_{s} \frac{2}{\pi}\left\{\frac{\pi}{2}-\frac{1}{2 i} \log \frac{\frac{x}{a} \cdot \frac{\sqrt{c^{2}-a^{2}}}{\sqrt{x^{2}-c^{2}}}+i}{\frac{\sqrt{c^{2}-a^{2}}}{\sqrt{x^{2}-c^{2}}}}-i\right\}, \quad|x|>c
\end{aligned}
$$

Since the electric displacement $D_{2}$ is finite, the coefficient of the singular term $\frac{x}{\sqrt{x^{2}-c^{2}}}$ must vanish. Hence we get

$$
\frac{a}{c}=\cos \left(\frac{\pi D_{2}^{\infty}}{2 D_{s}}\right)
$$

This result is completely similar with the Dugdale model. Thus, we have

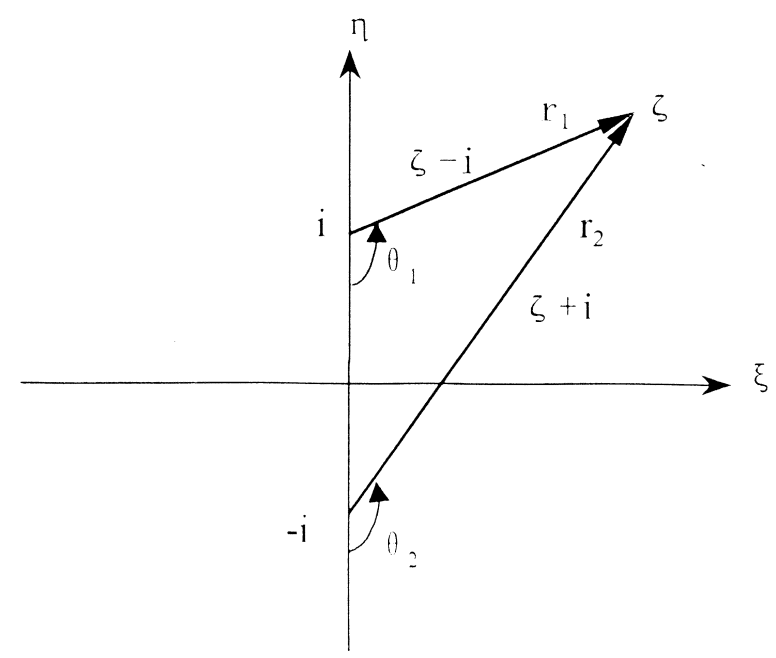

Fig. 2. Calculation scheme of complex function $\log \frac{\zeta+i}{\zeta-i}$. 


$$
\begin{aligned}
& D_{2}=\frac{2}{\pi} D_{s}\left\{\frac{\pi}{2}-\frac{1}{2 i} \log \frac{\frac{x}{a} \cdot \frac{\sqrt{c^{2}-a^{2}}}{\sqrt{x^{2}-c^{2}}}+i}{\frac{x}{a} \cdot \frac{\sqrt{c^{2}-a^{2}}}{\sqrt{x^{2}-c^{2}}}-i}\right\}-D_{2}^{\infty} \\
& =\frac{2}{\pi} D_{s}\left\{\frac{\pi}{2}-\frac{1}{2}\left(\theta_{2}-\theta_{1}\right)\right\}-D_{2}^{\infty}, \quad x>c, y=0
\end{aligned}
$$

The total electric displacement $\left(D_{2}\right)_{\text {total }}$ is

$$
\left(D_{2}\right)_{\mathrm{total}}=\frac{2}{\pi} D_{s} \arctan \left(\frac{x}{a} \cdot \frac{\sqrt{c^{2}-a^{2}}}{\sqrt{x^{2}-c^{2}}}\right), \quad x>c, y=0
$$

Now we consider the stress field ahead of the crack tip. From Eqs. (25), (29) and (38), it follows

$$
\begin{aligned}
& \boldsymbol{t}(x)=\boldsymbol{h}^{+}(x)+\boldsymbol{h}^{-}(x) \\
& t_{i}(x)=\Lambda_{i k}\left(g_{k}^{+}(x)+g_{k}^{-}(x)\right)+\Lambda_{i 4}\left(g_{4}^{+}(x)+g_{4}^{-}(x)\right) \\
& =\Lambda_{i k}^{*}\left(g_{k}^{+}(x)+g_{k}^{-}(x)\right)+\Lambda_{i 4}\left\{D_{2}^{\infty}\left(f^{\prime}{ }_{c}^{+}(x)+f^{\prime}{ }_{c}^{-}(x)\right)+D_{s}\left[g_{0}^{+}(x)+g_{0}^{-}(x)\right]\right\} / \Lambda_{44} \\
& =2 T_{i}^{*} f_{0}^{\prime}(x)+\Lambda_{i 4}\left\{D_{s}-D_{2}^{\infty}\right\} / \Lambda_{44} \\
& =T_{i}^{*} \frac{x}{\sqrt{x^{2}-a^{2}}}-T_{i}+\Lambda_{i 4} D_{s} / \Lambda_{44}, \quad a<x<c
\end{aligned}
$$

The total traction is

$$
\left(t_{i}(x)\right)_{\mathrm{total}}=\left(\sigma_{2 i}\right)_{\mathrm{total}}=T_{i}^{*} \frac{x}{\sqrt{x^{2}-a^{2}}}+\Lambda_{i 4} D_{s} / \Lambda_{44}, \quad a<x<c
$$

Obviously, this traction field can be characterized by the stress intensity factors:

$$
\begin{aligned}
& K_{\mathrm{II}}=\sqrt{\pi a}\left(\sigma_{12}^{\infty}-\frac{\Lambda_{14}}{\Lambda_{44}} D_{2}^{\infty}\right) \\
& K_{\mathrm{I}}=\sqrt{\pi a}\left(\sigma_{22}^{\infty}-\frac{\Lambda_{24}}{\Lambda_{44}} D_{2}^{\infty}\right) \\
& K_{\mathrm{III}}=\sqrt{\pi a}\left(\sigma_{23}^{\infty}-\frac{\Lambda_{34}}{\Lambda_{44}} D_{2}^{\infty}\right)
\end{aligned}
$$




\subsection{Crack perpendicular to the poling axis}

Fig. 1 shows a finite crack perpendicular to the poling axis. The matrix $\boldsymbol{H}$ has the following structure

$$
\boldsymbol{H}=\left[\begin{array}{cccc}
\frac{2}{C_{L}} & 0 & 0 & 0 \\
0 & \frac{2}{C_{T}} & 0 & \frac{2}{e} \\
0 & 0 & \frac{2}{C_{A}} & 0 \\
0 & \frac{2}{e} & 0 & -\frac{2}{\varepsilon}
\end{array}\right]
$$

The elements can be calculated numerically.

The matrix $\boldsymbol{\Lambda}$ is

$$
\Lambda=\left[\begin{array}{llll}
\frac{C_{L}}{2} & 0 & 0 & 0 \\
0 & \frac{C_{T}}{2 \rho_{0}} & 0 & \frac{C_{T}}{2 \rho_{0}} \frac{\varepsilon}{e} \\
0 & 0 & \frac{C_{A}}{2} & 0 \\
0 & \frac{C_{T}}{2 \rho_{0}} \frac{\varepsilon}{e} & 0 & -\frac{\varepsilon}{2 \rho_{0}}
\end{array}\right]
$$

where

$$
\rho_{0}=1+\frac{C_{T} \varepsilon}{e^{2}}
$$

Substituting above equation into Eq. (52), it follows

$$
\begin{aligned}
& K_{\mathrm{II}}=\sqrt{\pi a} \sigma_{12}^{\infty} \\
& K_{\mathrm{I}}=\sqrt{\pi a}\left(\sigma_{22}^{\infty}+\frac{C_{T}}{e} D_{2}^{\infty}\right) \\
& K_{\mathrm{III}}=\sqrt{\pi a} \sigma_{23}^{\infty}
\end{aligned}
$$

For our situation, the infinite plate only subjects to $\sigma_{11}^{\infty}, \sigma_{22}^{\infty}, D_{1}^{\infty}, D_{2}^{\infty}$ loading, hence we have

$$
\begin{aligned}
& K_{\mathrm{II}}=K_{\mathrm{III}}=0 \\
& K_{\mathrm{I}}=\sqrt{\pi a}\left(\sigma_{22}^{\infty}+\frac{C_{T}}{e} D_{2}^{\infty}\right)
\end{aligned}
$$

Since the electric displacements are finite and the stress components have the singularity ahead of the 
crack tip. Hence, use of the following local fracture criterion is reasonable.

$$
K_{\mathrm{I}}=K_{\mathrm{Ic}}
$$

Thus we obtain

$$
\left(\sigma_{22}^{\infty}\right)_{\mathrm{f}}=\sigma_{\mathrm{f} 0}-\frac{C_{T}}{e} D_{2}^{\infty}
$$

where

$$
\sigma_{\mathrm{f} 0}=\frac{K_{\mathrm{Ic}}}{\sqrt{\pi a}}
$$

Eq. (59) indicates that the critical failure stress is decreased linearly with applied electric field for a crack perpendicular to the poling axis. This result is consistent with the experiment result by Park and Sun (1995) and the result given by Gao et al. (1997).

\subsection{Crack parallel to poling axis}

Fig. 3 shows a finite crack parallel to the poling axis. Matrices $\boldsymbol{H}$ and $\boldsymbol{\Lambda}$ are

$$
\boldsymbol{H}=\left[\begin{array}{cccc}
\frac{2}{C_{T}} & 0 & 0 & \frac{2}{e} \\
0 & \frac{2}{C_{L}} & 0 & 0 \\
0 & 0 & \frac{2}{C_{A}} & 0 \\
\frac{2}{e} & 0 & 0 & -\frac{2}{\varepsilon}
\end{array}\right]
$$

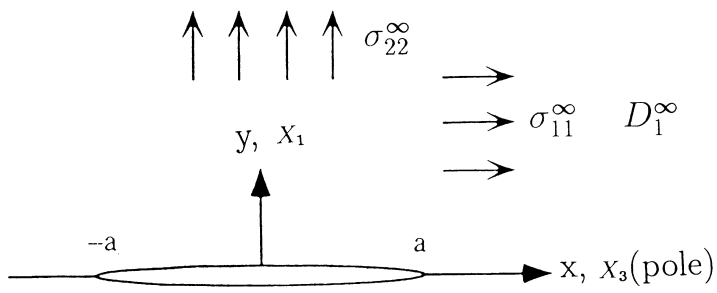

Fig. 3. A crack parallel to the poling axis. 


$$
\boldsymbol{\Lambda}=\left[\begin{array}{llll}
\frac{C_{T}}{2 \rho_{0}} & 0 & 0 & \frac{C_{T}}{2 \rho_{0}} \frac{\varepsilon}{e} \\
0 & \frac{C_{L}}{2} & 0 & 0 \\
0 & 0 & \frac{C_{A}}{2} & 0 \\
\frac{C_{T}}{2 \rho_{0}} \frac{\varepsilon}{e} & 0 & 0 & -\frac{\varepsilon}{2 \rho_{0}}
\end{array}\right]
$$

where $\rho_{0}=1+\frac{C_{T} \varepsilon}{e^{2}}$. Thus, we obtain

$$
\begin{aligned}
& K_{\mathrm{II}}=\sqrt{\pi a}\left(\sigma_{12}^{\infty}+\frac{C_{T}}{e} D_{2}^{\infty}\right) \\
& K_{\mathrm{I}}=\sqrt{\pi a} \sigma_{22}^{\infty} \\
& K_{\mathrm{III}}=\sqrt{\pi a} \sigma_{23}^{\infty}
\end{aligned}
$$

This result clearly shows that the applied electric field $D_{1}^{\infty}$ has no effect on the stress intensity factors. The applied electric field $D_{2}^{\infty}$, only has the contribution to the stress intensity factor $K_{\text {II }}$. If the infinite plate only subjects to the remote loadings $\sigma_{22}^{\infty}, D_{1}^{\infty}$, we obtain

$$
\begin{aligned}
& K_{\mathrm{II}}=K_{\mathrm{III}}=0, \quad K_{\mathrm{I}}=\sigma_{22}^{\infty} \sqrt{\pi a} \\
& \left(\sigma_{22}^{\infty}\right)_{\mathrm{f}}=\frac{K_{\mathrm{Ic}}}{\sqrt{\pi a}}
\end{aligned}
$$

Hence the failure stress $\left(\sigma_{22}^{\infty}\right)_{\mathrm{f}}$ is not effected by the applied electric field $D_{1}^{\infty}$.

\section{Analysis of fracture stress of piezoelectric ceramics with sharp notch}

The fracture tests for piezoelectric ceramics PZT-4 were performed by Park and Sun (1995). Fig. 4 shows the dimensions of their compact tension specimen. The poling direction was along the axis of 19.1 $\mathrm{mm}$ dimension. A notch of length $10.5 \mathrm{~mm}$ was introduced by cutting with a $0.46 \mathrm{~mm}$ thick diamond wheel perpendicular to the poling direction. Then the notch was further sharpened by a sharp razor blade with diamond abrasive. Hence, each peace of the compact tension specimens contains a sharp notch not a sharp crack. But the analyses given in Sections 2 and 3 are only suitable for the sharp crack. In order to analyse the experimental results by Park and Sun (1995) it is necessary to evaluate the stress fields ahead of the tip of a sharp notch. A program now is carried on for determining the stress fields ahead of the notch tip in real geometry of the compact tension specimens of piezoelectric ceramics PZT-4 by means of finite element method. Here, we only discuss an approximate analysis. Fig. 5 shows a centred sharp notch in an infinite piezoelectric ceramics. The half of the centred sharp notch has the same dimension and geometry as that of the sharp notch in the compact tension specimen. If the sharp notch is treated as a sharp crack, the stress intensity factors and the electric displacement intensity factors can be calculated based on finite element method. Table 1 shows the calculation results for the compact tension specimens of the piezoelectric ceramics PZT-4 given by one referee. From Table 1, one 


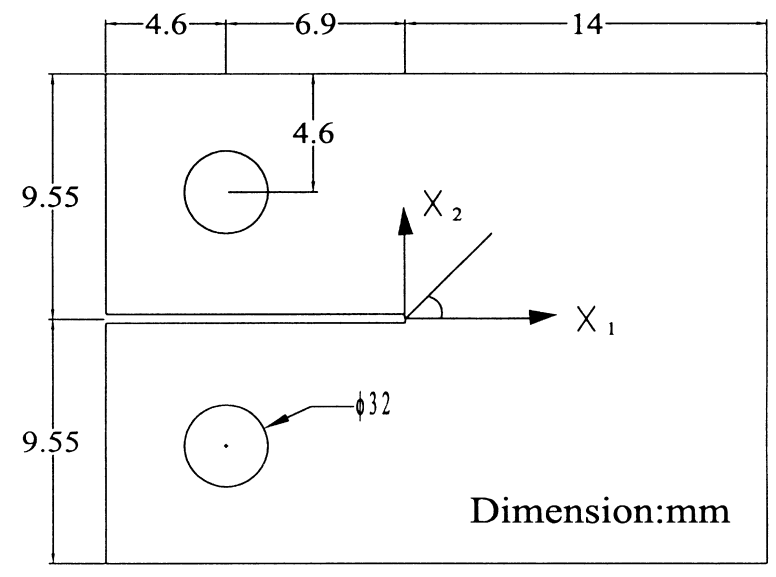

Fig. 4. A compact tension specimen of piezoelectric ceramics.

can get following formulae for the stress intensity factor $K_{I}$ and the electric displacement intensity factor $K_{D}$ :

$$
\begin{aligned}
& K_{I}=\frac{K_{I}^{(1)} P}{P_{0}}+\frac{K_{I}^{(2)} E}{E_{0}} \\
& K_{D}=\frac{K_{D}^{(1)} P}{P_{0}}+\frac{K_{D}^{(2)} E}{E_{0}}
\end{aligned}
$$

where $P_{0}=94.0 \mathrm{~N}, E_{0}=1.0 \mathrm{MV} / \mathrm{m}, K_{I}^{(1)}=0.81 \mathrm{MN} / \mathrm{m}^{3 / 2}, K_{I}^{(2)}=0.0406 \mathrm{MN} / \mathrm{m}^{3 / 2}, K_{D}^{(1)}=2.0 \times 10^{-4}$ $\mathrm{C} / \mathrm{m}^{3 / 2}$ and $K_{D}^{(2)}=14.4 \times 10^{-4} \mathrm{C} / \mathrm{m}^{3 / 2}$.

The following equivalent principal is introduced in the present analysis: the crack initiation and propagation from the tip of the sharp notch in the infinite piezoelectric ceramics will occur, if this sharp notch is treated as a sharp crack and subjected to the same stress intensity factors and electric displacement intensity factors as that of the compact tension specimen. The stress intensity factors and electric displacement intensity factors for a centred crack in an infinite piezoelectric ceramics are given by

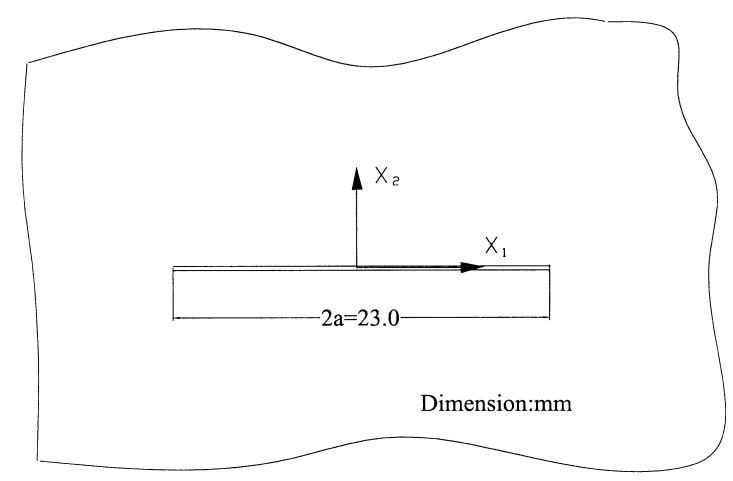

Fig. 5. A centred sharp notch in an infinite piezoelectric ceramics. 


$$
\begin{aligned}
& K_{\mathrm{II}}=\sqrt{\pi a} \sigma_{12}^{\infty} \\
& K_{\mathrm{I}}=\sqrt{\pi a} \sigma_{22}^{\infty} \\
& K_{\mathrm{III}}=\sqrt{\pi a} \sigma_{23}^{\infty} \\
& K_{D}=\sqrt{\pi a} D_{2}^{\infty}
\end{aligned}
$$

Since the stress intensity factors $K_{\mathrm{II}}$ and $K_{\mathrm{III}}$ equal to zero for the compact specimens, hence we have $\sigma_{12}^{\infty}=\sigma_{23}^{\infty}=0$. The remote stress $\sigma_{22}^{\infty}$ and the remote electric displacement $D_{2}^{\infty}$ applied on the infinite piezoelectric ceramics with a centred crack can be expressed as follows

$$
\begin{aligned}
& \sigma_{22}^{\infty}=\frac{K_{I}}{\sqrt{\pi a}} \\
& D_{2}^{\infty}=\frac{K_{D}}{\sqrt{\pi a}}
\end{aligned}
$$

Now the same remote stress $\sigma_{22}^{\infty}$ and the remote electric displacement $D_{2}^{\infty}$ apply on the infinite piezoelectric ceramics with a centred sharp notch. In order to get an analytical solution, the original centred sharp notch is approximately treated as an elliptic notch with the same length and the same curvature at the sharp tip of the notch as that of the original centred sharp notch. Let us discuss the fracture problem of the infinite piezoelectric ceramics with a sharp elliptic notch and analyse the effect of the remote electric displacement field on the fracture stress. Following the work by Chung and Ting (1996) the maximum stress $\left(\sigma_{22}\right)_{\max }$ at the sharp tip of an elliptic notch can be expressed as:

$$
\left(\sigma_{22}\right)_{\max }=F_{I} \sigma_{22}^{\infty}+F_{D} D_{2}^{\infty}
$$

where the coefficient $F_{I}$ is the stress concentrate factor and the coefficient $F_{D}$ represents the effect factor of the remote electric displacement $D^{\infty_{2}}$ on the stress $\left(\sigma_{22}\right)_{\max }$. The detail formulas for determining the coefficients $F_{I}$ and $F_{D}$ are given in Appendix A. The critical maximum stress criterion is adopted in the present analysis. Hence the fracture stress $\left(\sigma_{22}^{\infty}\right)_{\mathrm{f}}$ is given by

$$
\left(\sigma_{22}^{\infty}\right)_{\mathrm{f}}=\frac{\sigma_{\mathrm{f}}-F_{D} D_{2}^{\infty}}{F_{I}}
$$

where $\sigma_{\mathrm{f}}$ is the fracture strength of the piezoelectric material.

Table 1

The stress intensity factor and the electric displacement intensity factor for compact specimen of PZT-4

\begin{tabular}{lcccc}
\hline No. & $E(\mathrm{MV} / \mathrm{m})$ & $P(\mathrm{~N})$ & $K_{I}\left(\mathrm{MN} / \mathrm{M}^{3 / 2}\right)$ & $K_{D}\left(10^{-4} \mathrm{C} / \mathrm{m}^{3 / 2}\right)$ \\
\hline 1 & -0.52 & 126.0 & 1.05 & -4.80 \\
2 & -0.26 & 108.0 & 0.90 & -1.45 \\
3 & 0.0 & 94.0 & 0.81 & 2.00 \\
4 & 0.26 & 78.9 & 0.70 & 5.41 \\
5 & 0.52 & 71.4 & 0.65 & 9.00 \\
6 & 1.06 & 68.1 & 0.63 & 16.7 \\
\hline
\end{tabular}




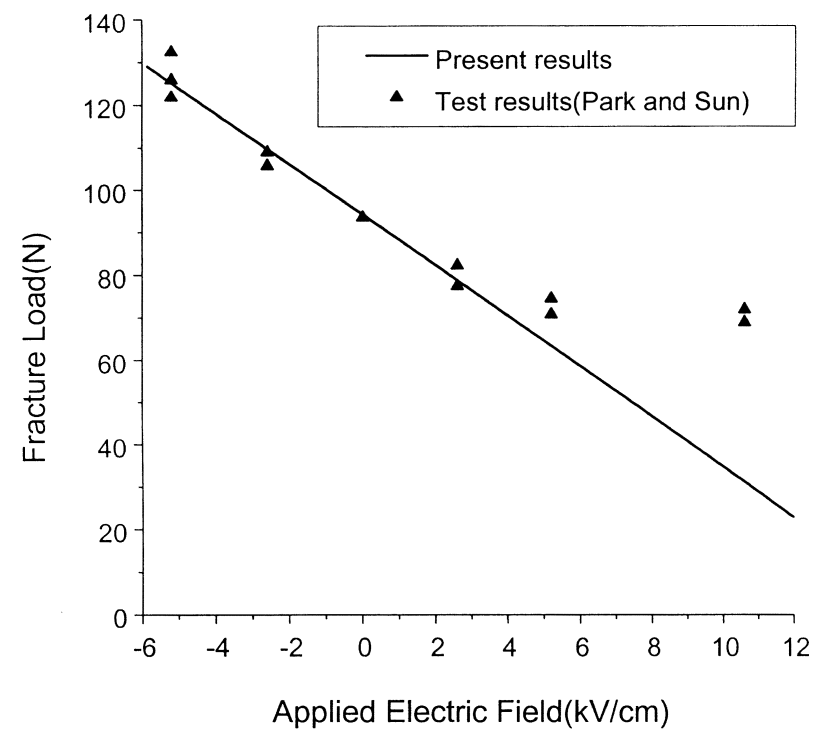

Fig. 6. Comparison of the present calculation results with the experiment results.

For a given $D_{2}^{\infty}$, one can get the critical stress $\left(\sigma_{22}^{\infty}\right)_{\mathrm{f}}$ from Eq. (70). Then the critical stress intensity factor $K_{I}$ and the critical electric displacement intensity factor $K_{D}$ can be obtained from Eq. (67). Substitute these results into the left-hand side of Eq. (66), one can get the critical load $P$ and the critical electric field strength $E$ for the compact tension specimens based on the equivalent principal.

The calculated results are shown in Fig. 6 alone with the experiment results by Park and Sun (1995). The material parameters and the dimensions of the sharp notch are taken from their work. The curvature $\rho$ at the sharp notch tip is chosen as $40 \mu \mathrm{m}$ and the corresponding fracture strength $\sigma_{\mathrm{f}}$ is taken as $192 \mathrm{MPa}$.

It is worth noting that the present calculation results are consistent with the experimental results by Park and Sun (1995) besides the data of $E>1 \mathrm{MV} / \mathrm{m}$. Since we do not know the exact value of the curvature $\rho$. Hence the calculation is also carried out for the case of the curvature $\rho=10 \mu \mathrm{m}$ and the corresponding fracture strength $\sigma_{\mathrm{f}}=380 \mathrm{MPa}$. The calculation results are nearly identical with the results for the case of $\rho=40 \mu \mathrm{m}$. It means that the load $P$ seems not sensitive to the curvature. But the curvature has significant effect on the fracture strength of the piezoelectric ceramics.

\section{Discussion}

The fully anisotropic analysis presented in this paper confirms the analysis given by Gao et al. (1997) which is based on a simplified electroelasticity formulation. The prediction of the strip electric saturation model broadly agrees with experimental observations. Vicker indentation technique has been used for producing cracking both perpendicular and parallel to the poling direction (Tobin and Pak, 1993; Virkar et al., 1991; Lynch et al., 1995). It has been revealed that cracks perpendicular to the poling axis are longer under a positive applied electric field and shorter under a negative applied electric field. It implies that the former has a higher fracture resistance and the latter has a lower fracture resistance.

A parallel investigation is to consider the effects of electric domain switch. Ferroelectric ceramics show strong nonlinearities at combined mechanical and electric loadings. The polarization switching 
may play an essential role in response to the complicated nonlinear phenomena. These phenomena have been modelled by Yang and Suo (1994), Lynch et al. (1995) and Zhu and Yang (1997) among others. A further study of the fully anisotropic analysis taking into account the effects of electric domain switching is needed. The electric saturation zone is assumed to concentrate on a line segment in front of the crack tip in this paper. A more realistic electric saturation model combining with electric domain switching is more interesting for a through understanding of fracture behaviors of ferroelectric ceramics.

\section{Acknowledgements}

The author is grateful for the joint supports by the National Natural Science Foundation of China (contract 19392300) and the Natural Science Foundation of Chinese Academy of Sciences (project KJ951-1-201). The author appreciates the valuable comments and the nice help given by referees. One referee provided me the calculated results of stress intensity factors and the electric displacement intensity factors for the compact specimens of piezoelectric ceramics PZT-4.

\section{Appendix A}

An ellipse $\Gamma$ with semi-axes $a$ and $b$ in the physical plane can be transformed into the unit circle in the mapping plane $\zeta$ by following mapping function

$$
z=\omega(\zeta)=R\left(\zeta+\frac{m}{\zeta}\right)
$$

where $R=(a+b) / 2, m=(a-b) /(a+b)$.

Consider the transformation

$$
z_{K}=c_{K} \zeta_{K}+\frac{d_{K}}{\zeta_{K}} \quad(K=1,2,3,4)
$$

where $c_{K}$ and $d_{K}$ are complex constants and $z_{K}=x_{1}+p_{K x_{2}}$. The constants $c_{K}$ and $d_{K}$ are chosen such that when $z$ on $\Gamma, \zeta_{K}$ is on the unit circle of the $\zeta$ plane. Hence, we have

$$
c_{K}=\frac{a-i p_{K} b}{2}, \quad d_{K}=\frac{a+i p_{K} b}{2}
$$

Follow Chung and Ting (1996) the generalized displacement $\boldsymbol{u}$ and the generalized stress function $\boldsymbol{\Phi}$ outside the $\Gamma$ can be expressed as

$$
\begin{aligned}
& \boldsymbol{U}=\left\{u_{j}, \phi\right\}=\boldsymbol{A} \boldsymbol{f}_{*}\left(z_{*}\right)+\bar{A} \overline{\boldsymbol{f}_{*}\left(z_{*}\right)} \\
& \boldsymbol{\Phi}=\left\{\Phi_{K}, K=1,2,3,4\right\}=\boldsymbol{B} \boldsymbol{f}_{*}\left(z_{*}\right)+\overline{\boldsymbol{B}} \overline{\boldsymbol{f}_{*}\left(z_{*}\right)}
\end{aligned}
$$

where

$$
\boldsymbol{f}_{*}\left(z_{*}\right)=\left\{f_{1}\left(z_{1}\right), f_{2}\left(z_{2}\right), f_{3}\left(z_{3}\right), f_{4}\left(z_{4}\right)\right\}
$$

Introduce a new transformation 


$$
z_{K}^{*}=c_{K} \zeta+\frac{d_{K}}{\zeta} \quad(K=1,2,3,4)
$$

All $z_{K}^{*}$ depends on a single variable $\zeta$.

Define a new function $\boldsymbol{F}(\zeta)$

$$
\begin{aligned}
& \boldsymbol{F}(\zeta)=\left\{F_{K}(\zeta), K=1,2,3,4\right\} \\
& F_{K}(\zeta)=f_{K}\left(z_{K}^{*}\right)=f_{K}\left(c_{K} \zeta+\frac{d_{K}}{\zeta}\right)
\end{aligned}
$$

Then the generalized displacement $\boldsymbol{u}$ and the generalized stress function $\boldsymbol{\Phi}$ on $\Gamma$ can be expressed as

$$
\begin{aligned}
& \boldsymbol{U}=\boldsymbol{A} \boldsymbol{F}(\zeta)+\overline{\boldsymbol{A}} \overline{\boldsymbol{F}(\zeta)} \\
& \boldsymbol{\Phi}=\boldsymbol{B} \boldsymbol{F}(\zeta)+\overline{\boldsymbol{B}} \overline{\boldsymbol{F}(\zeta)}
\end{aligned}
$$

Inside the notch, the stress fields vanish and the electric fields and the electric displacement fields are uniform. Hence we have

$$
\boldsymbol{u}^{0}=x_{1} \gamma_{1}^{0}+x_{2} \gamma_{2}^{0}, \quad \boldsymbol{\Phi}^{0}=x_{1} \boldsymbol{t}_{2}^{0}-x_{2} \boldsymbol{t}_{1}^{0}
$$

where

$$
\gamma_{1}^{0}=\left\{\gamma_{11}^{0}, \gamma_{21}^{0}, 2 \gamma_{31}^{0},-E_{1}^{0}\right\}, \quad \gamma_{2}^{0}=\left\{\gamma_{12}^{0}, \gamma_{22}^{0}, 2 \gamma_{32}^{0},-E_{2}^{0}\right\}
$$

and

$$
\boldsymbol{t}_{1}^{0}=\left\{0,0,0, D_{1}^{0}\right\}, \quad \boldsymbol{t}_{2}^{0}=\left\{0,0,0, D_{2}^{0}\right\}
$$

in which $D_{1}^{0}=\varepsilon_{0} E_{1}^{0}$ and $D_{2}^{0}=\varepsilon_{0} E_{2}^{0}, \varepsilon_{0}$ is the dielectric constant of the air. Eq. (9) can be written as

$$
\boldsymbol{u}^{0}=2 \operatorname{Re}\left(\frac{\gamma_{1}^{0}-i \gamma_{2}^{0}}{2} z\right), \quad \boldsymbol{\Phi}^{0}=2 \operatorname{Re}\left(\frac{\boldsymbol{t}_{2}^{0}+\boldsymbol{i t}_{1}^{0}}{2} z\right)
$$

The continuity of the generalized displacement $\boldsymbol{u}$ and the generalized stress function $\boldsymbol{\Phi}$ on $\Gamma$ can be expressed as

$$
\begin{gathered}
\boldsymbol{U}=\boldsymbol{A} \boldsymbol{F}(\zeta)+\overline{\boldsymbol{A}} \overline{\boldsymbol{F}(\zeta)}=2 \operatorname{Re}\left(\frac{\boldsymbol{\gamma}_{1}^{0}-i \gamma_{2}^{0}}{2} z\right) \\
\boldsymbol{\Phi}=\boldsymbol{B} \boldsymbol{F}(\zeta)+\overline{\boldsymbol{B}} \overline{\boldsymbol{F}(\zeta)}=2 \operatorname{Re}\left(\frac{\boldsymbol{t}_{2}^{0}+i i_{1}^{0}}{2} z\right)
\end{gathered}
$$

The solution of Eq. (A13b) is

$$
\boldsymbol{B F}=R \varepsilon_{0} \beta_{0} \boldsymbol{I}_{4} \frac{1}{\zeta}+\boldsymbol{\Gamma}_{1} \zeta+\boldsymbol{\Gamma}_{2} \frac{1}{\zeta}, \quad \zeta \Omega^{+}
$$

where $\boldsymbol{\Gamma}_{1}=\boldsymbol{B} \boldsymbol{C} \boldsymbol{q}$ and $\boldsymbol{\Gamma}_{2}=-\overline{\boldsymbol{\Gamma}}_{1}, \boldsymbol{q}$ is an unknown column vector, needed to be determined and $\boldsymbol{C}$ is a matrix 


$$
\boldsymbol{C}=\operatorname{diag}\left\{C_{1}, C_{2}, C_{3}, C_{4}\right\}
$$

and $\boldsymbol{I}_{4}$ is a column vector

$$
\boldsymbol{I}_{4}=\{0,0,0,1\}
$$

Substitute Eq. (A14) into Eq. (A13a), one obtains

$$
\overline{\boldsymbol{A B}}{ }^{-1} I_{4} \varepsilon_{0} \bar{\beta}_{0}-\alpha_{0}=\left(\overline{A B^{-1}}-\boldsymbol{A B}{ }^{-1}\right) \boldsymbol{B C q} / R
$$

in which

$$
\boldsymbol{\alpha}_{0}=\frac{\boldsymbol{\gamma}_{1}^{0}-i \gamma_{2}^{0}}{2}+m \frac{\gamma_{1}^{0}+i \gamma_{2}^{0}}{2}
$$

and

$$
\beta_{0}=\frac{E_{2}^{0}-i E_{1}^{0}}{2}+m \frac{E_{2}^{0}+i E_{1}^{0}}{2}
$$

In the infinity, the function $f_{K}\left(z_{K}\right)$ approaches to

$$
f_{K}\left(z_{K}\right)=q_{K} z_{K}=q_{K}\left(x_{1}+p_{K} x_{2}\right)
$$

In the infinity, we have

$$
\begin{aligned}
& \boldsymbol{f}_{*}\left(z_{*}\right)=\boldsymbol{q} x_{1}+\boldsymbol{P} \boldsymbol{q} x_{2} \\
& \boldsymbol{\Phi}=2 \operatorname{Re}\left\{\boldsymbol{B} \boldsymbol{f}_{*}\left(z_{*}\right)\right\}=2 \operatorname{Re}\left\{\boldsymbol{B}\left(\boldsymbol{q} x_{1}+\boldsymbol{P} \boldsymbol{q} x_{2}\right)\right\}
\end{aligned}
$$

where $\boldsymbol{P}$ is the matrix

$$
\boldsymbol{P}=\operatorname{diag}\left\{p_{1}, p_{2}, p_{3}, p_{4}\right\}
$$

From Eq. (A18), it follows

$$
\begin{aligned}
& B \boldsymbol{B}+\overline{\boldsymbol{B q}}=\boldsymbol{t}_{2}^{\infty} \\
& \boldsymbol{B P q}+\overline{\boldsymbol{B P q}}=-\boldsymbol{t}_{1}^{\infty}
\end{aligned}
$$

$\boldsymbol{t}_{1}^{\infty}$ and $\boldsymbol{t}_{2}^{\infty}$ are the remote generalized tractions. One can easily obtained the column vector $\boldsymbol{q}$ from Eq. (19) for any given $\boldsymbol{t}_{1}^{\infty}$ and $\boldsymbol{t}_{2}^{\infty}$.

Using Eq. (14), the function $F_{K}(\zeta)$ can be expressed as

$$
F_{K}(\zeta)=f_{K}\left(c_{K} \zeta+d_{K} \frac{1}{\zeta}\right)=\xi_{K} \zeta+\eta_{K} \frac{1}{\zeta}
$$

where

$$
\begin{aligned}
& \boldsymbol{\xi}=\left\{\xi_{1}, \xi_{2}, \xi_{3}, \xi_{4}\right\}=\boldsymbol{C} \boldsymbol{q} \\
& \boldsymbol{\eta}=\left\{\eta_{1}, \eta_{2}, \eta_{3}, \eta_{4}\right\}=\boldsymbol{B}^{-1}\left\{R \varepsilon_{0} \beta_{0} \boldsymbol{I}_{4}+\boldsymbol{\Gamma}_{2}\right\}
\end{aligned}
$$


The variable $\zeta$ can be expressed in term of $z_{K}^{*}$

$$
\zeta=\frac{z_{K}^{*}+\sqrt{z_{K}^{* 2}-4 c_{K} d_{K}}}{2 c_{K}}
$$

Hence the function $f_{K}\left(z_{K}^{*}\right)$ can be written as

$$
f_{K}\left(z_{K}^{*}\right)=\xi_{K} \frac{z_{K}^{*}+\sqrt{z_{K}^{* 2}-4 c_{K} d_{K}}}{2 c_{K}}+\eta_{K} \frac{z_{K}^{*}-\sqrt{z_{K}^{* 2}-4 c_{K} d_{K}}}{2 d_{K}}
$$

and

$$
\frac{\mathrm{df}_{\mathrm{K}}\left(z_{K}\right)}{\mathrm{dz}}=\frac{1}{\sqrt{z_{K}^{2}-4 c_{K} d_{K}}}\left[\xi_{K} \frac{z_{K}+\sqrt{z_{K}^{2}-4 c_{K} d_{K}}}{2 c_{K}}-\eta_{K} \frac{z_{K}-\sqrt{z_{K}^{2}-4 c_{K} d_{K}}}{2 d_{K}}\right]
$$

when $\zeta=1$

$$
\frac{\mathrm{d} f_{K}\left(z_{K}\right)}{\mathrm{d} z_{K}}=\xi_{K} \frac{1+i a / p_{K} b}{2 c_{K}}+\eta_{K} \frac{1-i a / p_{K} b}{2 d_{K}}
$$

The maximum stress $\left(\sigma_{22}\right)_{\max }$ on the tip of the sharp elliptic notch can be determined by

$$
\left(\sigma_{22}\right)_{\max }=2 \operatorname{Re}\left\{\sum_{K=1}^{4} B_{2 K} f_{K}^{\prime}\left(z_{K}\right)\right\}=\operatorname{Re}\left\{\sum_{K=1}^{4} B_{2 K}\left\{\left[\frac{\xi_{K}}{c_{K}}+\frac{\eta_{K}}{d_{K}}\right]+\left[\frac{\xi_{K}}{c_{K}}-\frac{\eta_{K}}{d_{K}}\right] \frac{i a}{p_{K} b}\right\}\right\}
$$

\section{References}

Chung, M.Y., Ting, T.C.T., 1996. Piezoelectric solid with an elliptic inclusion or hole. I. J. Solids and Structure 33, $3343-3361$.

Deeg, W.F.J., 1980. The analysis of dislocation, crack, and inclusion problems in piezoelectric solids. Ph.D. Thesis, Stanford University.

Dunn, M.L., 1980. The effects of crack face boundary conditions on the fracture mechanics. Eng. Frac. Mech 48, 25-39.

Gao, H.J., Zhang, T.Y., Tong, P., 1997. Local and global energy release rates for an electrically yielded crack in a piezoelectric ceramic. J. Mech. Phys. Solids 45, 491-510.

Kumar, S., Singh, R.N., 1996. Crack propagation in piezoelectric materials under combined mechanical and electrical loadings. Acta Mater 44, 173-200.

Lynch, C.S., Yang, W., Collier, L., Suo, Z., McMeeking, R.M., 1995. Electric field induced cracking in ferroelectric ceramics. Ferroelectrics 166, 11-30.

McMeeking, R.M., 1989. Electrostrictive forces near crack like flaws. J. Appl. Phys 40, 615-627.

Pak, Y.E., Herrmann, G., 1986. Conservation laws and the material momentum tensor for the elastic dielectric. Int. J. Engng., Sci 24, 1365-1374.

Pak, Y.E., 1990. Crack extension force in a piezoelectric material. J. Appl. Mech 57, 647-653.

Pak, Y.E., Tobin, A., 1993. On electric field effects in fracture of piezoelectric materials, Mechanics of Electromagnetic Materials and Structures, AMD-vol. 161/MD-vol. 42, ASME.

Park, S., Sun, C.T., 1995. Fracture criteria for piezoelectric materials. J. Am. Ceram. Soc 78, 1475-1480.

Parton, V.Z., 1976. Fracture mechanics of piezoelectric materials. Acta Astronautica 3, 671-683.

Sosa, H., 1992. On the fracture mechanics of piezoelectric solids. Int. J. Solids Structures 29, 2613-2622.

Suo, Z., Kuo, C.M., Barnett, D.M., Willis, J.R., 1992. Fracture mechanics for piezoelectric ceramics. J. Mech. Phys. Solids 40, 739-765.

Suo, Z., 1993. Models for breakdown-resistant dielectric and ferroelectric ceramics. J. Mech. Phys. Solids 41, 1155-1176.

Tobin A.G., Pak Y.E., 1993. Effects of electric field on fracture of piezoelectric ceramics. Proc. SPIE, Smart Struc. Mater. 1916, 78-86. 
Virkar, A.V., Jue, J.F., Smith, P., Mehta, K., Prettyman, K., 1991. The role of ferroelasticity in toughening of brittle materials. Phase Transitions 35, 27-46.

Yang, W., Suo, Z., 1994. Cracking in ceramic actuators caused by electrostriction. J. Mech. Phys. Solids 42, 649-663.

Zhang, T.Y., Hack, J.E., 1992. Mode III cracks in piezoelectric materials. J. Appl. Phys 71, 5865-5870.

Zhang, T.Y., Tong, P., 1996. Fracture mechanics for a mode III crack in a piezoelectric material. Int. J. Solids \& Struct 33, $343-$ 359.

Zhu, T., Yang, W., 1997. Toughness variation of ferroelectrics by polarization switch under non-uniform electric field. Acta Mater 45, 4695-4702. 